\title{
Using CRISPR-Cas systems as antimicrobials
}

David Bikard ${ }^{1} \&$ Rodolphe Barrangou ${ }^{2}$

${ }^{1}$ Synthetic Biology Group, Microbiology Department, Institut Pasteur, Paris 75015, France, david.bikard@pasteur.fr

${ }^{2}$ Department of Food, Processing and Nutritional Sciences, North Carolina State University, NC, rbarran@ncsu.edu

Corresponding authors: David Bikard \& Rodolphe Barrangou

\section{Highlights}

- Self-targeting by CRISPR-Cas systems is typically lethal in bacteria

- CRISPR-Cas systems can be repurposed to drive programmed bacterial death

- CRISPR arrays and Cas nucleases can be delivered to target organisms using phages

- There is a need to engineer delivery vectors to enable CRISPR-based antimicrobials 


\section{Abstract}

2 Although CRISPR-Cas systems naturally evolved to provide adaptive immunity in bacteria and

3 archaea, Cas nucleases can be co-opted to target chromosomal sequences rather than invasive

4 genetic elements. While genome editing is the primary outcome of self-targeting using CRISPR-based

5 technologies in eukaryotes, self-targeting by CRISPR is typically lethal in bacteria. Here, we discuss

6 how DNA damage introduced by Cas nucleases in bacteria can efficiently and specifically lead to

7 plasmid curing or drive cell death. Specifically, we discuss how various CRISPR-Cas systems can be

8 engineered and delivered using phages or phagemids as vectors. These principles establish CRISPR-

9 Cas systems as potent and programmable antimicrobials, and open new avenues for the

10 development of CRISPR-based tools for selective removal of bacterial pathogens and precise

11 microbiome composition alteration. 
Clustered regularly interspaced short palindromic repeats (CRISPR) together with CRISPR-associated (Cas) proteins constitute the adaptive immune system of prokaryotes [1]. Over the past 10 years, they have captured the attention of the scientific community in many ways, spanning their role in driving the co-evolutionary interplay between bacteria and their viruses, and the development of CRISPR-based technologies [2-4]. Most of the applications rely on the discovery of programmable RNA-guided nucleases among the CRISPR associated (Cas) proteins. By designing the sequence of a small CRISPR guide RNA, scientists are readily able to direct these nucleases in an extremely precise manner to virtually any genomic locus of interest to drive a variety of molecular outcomes.

The diversity of CRISPR-Cas systems has been reviewed elsewhere [5]. Here, we will mostly focus on type I and II CRISPR-Cas systems, which have been used to selectively kill bacteria. In nature, Type I systems are the most widespread and hinge on a multi-protein effector complex, the CRISPRassociated complex for antiviral defense (CASCADE)[6], which uses the Cas3 exonuclease for processing of invasive DNA [7]. This is in contrast to Type II systems which rely on the potent signature effector endonuclease Cas9 to generate double stranded DNA breaks [8-10]. Conveniently, the machinery of CRISPR-Cas systems can be ported as a two component system comprising the protein Cas9 and a single guide RNA (sgRNA) mimicking the dual native crRNA:tracrRNA complex typically found in nature [10]. DNA breaks generated by Cas9 can be repaired, leading to precise alteration of the DNA sequence at the exact site of cleavage, essentially editing DNA with accuracy. In addition to sequence alteration, other Cas9-based technologies have recently been developed to precisely control gene expression, modify the epigenetic state of a sequence, fluorescently tag genomic loci, and carry out high-throughput genetic screens $[2,11,12]$. Even though most of the ongoing efforts focus on eukaryotic applications of CRISPR-based technologies, CRISPR-Cas systems afford tremendous opportunities in bacteria, where either endogenous or heterologous CRISPR-Cas systems can be readily repurposed for a variety of applications, including genome editing and control of gene expression [13-16]. We focus here on the use of both type I \& II CRISPR-Cas systems as programmable antimicrobials. We discuss how they can be readily directed to target undesirable sequences such as antibiotic resistance and virulence genes, with the purpose of eradicating pathogenic bacteria, or as a means to destroy the undesirable plasmids they occasionally carry [17].

\section{Self-targeting by CRISPR-Cas systems in nature}

While CRISPR-Cas systems are functionally designed to target invasive nucleic acids, they can occasionally sample chromosomal DNA from their native host. Indeed, while most CRISPR spacers match phage and plasmid DNA, some can show homology to chromosomal sequences [18]. An early report from Sorek and colleagues actually noted that in nature some CRISPR-Cas systems carry 
spacers that perfectly match sequences encoded on the host chromosome [19]. While some of these self-targeting spacers match prophages and other mobile elements such as transposons, others are found to match genes surprisingly belonging to the core genome. Nonetheless, in almost all of these cases, the authors were able to identify clues that the CRISPR-Cas system has been somehow inactivated by mutations in the cas genes or CRISPR array, or mutations altering the targeted sequences. Mutations in the target sequence that block CRISPR immunity typically occur in the protospacer-adjacent motif (PAM) (Horvath et al., 2008), or within the seed sequence [20,21], which encompasses the cleavage site and drives the formation of the crRNA:targetDNA R-loop for cleavage [22]. A model was thus proposed where the CRISPR-Cas systems sometimes capture self-targeting spacers "by mistake" and can only survive such events if the system is functionally inactivated. A study investigating the spacer acquisition process in the Streptococcus thermophilus model system showed that acquisition from chromosomal sequences can occur, but established that this autoimmune sampling is rare, and that when it does occur, these genotypes do not remain in the bacterial population [23]. Specifically, the authors were able to detect acquisition from chromosomal sequences at a frequency of $0.04 \%$ (120 of 443,871 acquired spacers), and showed that these selftargeting events could only be detected at one time point, and disappeared within a day from the population, presumably because acquisition of self-targeting spacers is lethal. Several mechanisms enable CRISPR-Cas systems to preferentially acquire spacers from foreign DNA, including primed adaptation [24] and acquisition from DNA fragments generated after the processing by exonucleases of double strand breaks, or phage DNA termini $[25,26]$. Despite these mechanisms, it is possible to detect lethal acquisition events in natural CRISPR-Cas systems [27].

\section{The primary outcome of self-targeting is cell death}

The first report of cell death induced by a CRISPR-Cas system targeting a sequence on the bacterial chromosome was published by Qimron and colleagues. In this study, they investigated the consequences of directing the native type I-E CRISPR-Cas system from Escherichia coli to an integrated lambda prophage [28]. They observed that inducing the CRISPR-Cas system led to the death of $98 \%$ of the cells in the population, and already speculated at the time that cell death was the result of chromosomal DNA degradation by the Cas enzymatic machinery. It was later shown that self-targeting by Type I systems efficiently killed bacteria regardless of the target location [29], and can lead to the excision of large pieces of DNA in the target region [30]. In Type I systems, the CASCADE complex binds the target and recruits the Cas3 exonuclease leading to extensive DNA degradation [31,32]. This is mechanistically different from Type II systems where Cas9 cleaves DNA endo-nucleolytically, by cutting target DNA exactly $3 \mathrm{nt}$ away from the $3^{\prime}$ edge of the targeted protospacer [8], using two nickase domains $[9,10]$. Both CRISPR-Cas types were nonetheless shown to have 
the ability to cure plasmids, or kill bacteria when reprogrammed to target the chromosome (Figure 1a) [33].

The ability to kill a population of bacteria based on its sequence using the type II system from $S$. pyogenes was used as a means to select for the introduction of mutations [14], providing the first evidence of CRISPR-mediated genome editing in bacteria. The type I systems from E. coli and Salmonella, as well as the type II system from $S$. thermophilus were also used to selectively eliminate even closely related organisms ( $99 \%$ genomic identity) by targeting unique sequences in a complex microbial population [29]. Across types and subtypes and organisms, investigators have generally observed that self-targeting using endogenous CRISPR-Cas systems in bacteria kills the large majority of the bacterial population, with killing efficiency ranging between 2 and 5 orders of magnitude of cell death, with single spacers.

\section{Causing DNA damage with Cas nucleases}

Most organisms, including bacteria, are regularly subjected to double stranded breaks and other types of DNA damage. Consequently, they have evolved complex DNA repair pathways that enable to maintain genomic integrity. One might thus wonder why CRISPR-Cas systems are so efficient at killing bacteria. In the case of type I systems, Cas3 possesses both ssDNA exonuclease and $3^{\prime}$ to $5^{\prime}$ helicase activities $[34,35]$. The introduction or activation of a self-targeting type I system leads to immediate cell death and efficient degradation of DNA up to $100 \mathrm{~kb}$ away from the target position in a few hours [32]. In type II systems however, Cas9 only introduces a double strand break (DSB). DSBs are recognized by exonucleases such as recBCD or addAB, which create ssDNA substrates for homology directed repair, via homologous recombination [36]. The consequences of Cas9 cleavage in the chromosome of $E$. coli was recently investigated in detail [37]. It was found that some crRNAs efficiently guide Cas9 to cut all copies of the chromosome simultaneously, making repair through recombination with a sister chromosome impossible and thus efficiently killing the cell. However, other crRNAs lead to less efficient targeting resulting in cell survival thanks to a continuous loop of cleavage and repair. The authors were able to block this phenomenon by expressing the Gam protein from bacteriophage Mu during Cas9 targeting. This protein binds to double stranded ends and blocks homologous recombination, leading to cell death regardless of the target choice. By controlling the outcome of CRISPR self-targeting, scientists can thus either edit genomes or induce cell death.

\section{Delivery}

While CRISPR self-targeting has been shown in principle to be a potent programmable antimicrobial, the main challenge for its repurposing to eradicate bacteria responsible for infectious disease is delivery to the target population, with both specificity and efficiency that would afford clinically- 
relevant efficacy. Several studies have shown how DNA encoding bactericidal proteins other than Cas nucleases can be delivered to bacterial populations using phage particles as vectors. The M13 phagemid was used to deliver various toxins or restriction enzymes to $E$. coli [38-40]. The Pf3 phage has also been used to a deliver a restriction enzyme, and successful treat a $P$. aeruginosa infection in mice [41].

Inspired by these early results, two studies, one in E. coli, the other in S. aureus, have provided a proof of concept for delivery of self-targeting CRISPR-Cas systems to pathogenic bacteria using phage capsids as delivery vectors $[12,42]$. In the first study, the M13 phagemid system was used to inject a genetic construct containing Cas9 and guide RNAs targeting various antibiotic resistance genes, namely bla NDM-1, blaSHV-18, and gyrAD87G. As expected, when the target gene was present in the chromosome, the outcome of Cas9 cleavage was efficient $E$. coli cell death. Interestingly, targeting a plasmid could also lead to cell death when the plasmid carried a toxin-antitoxin system, but otherwise yielded plasmid curing. In the second study, the authors constructed a phagemid based on phage phiNM1 by cloning its packaging site on a plasmid carrying the CRISPR-Cas system, to target $S$. aureus. This phagemid was used to target various antibiotic resistance genes, as well as virulence factors carried either on plasmids or on the chromosome. Both studies demonstrated the possibility of using CRISPR-Cas systems to specifically eliminate a target bacterial genotype in a mixed population, both in vitro and in vivo, using a wax worm infection model in the first case and a mouse skin colonization model in the other.

Delivering the CRISPR-Cas system to the majority of a target population in a complex environment, where the disease agent might be present in only small amounts, represents a big challenge. Qimron and colleagues proposed an elegant strategy to give a fitness advantage to bacteria that receive the CRISPR-Cas system [43]. In a first step they delivered a CRISPR-Cas system carrying a set of spacers targeting beta-lactam resistance genes. This presumably re-sensitizes $E$. coli cells that carry plasmids with the targeted sequences to the antibiotic. In a second step, they selected bacteria that received this system by using a lytic T7 phage modified to carry target sequences matching the CRISPR spacers. Only cells that carry an active CRISPR-Cas systems with the proper anti-beta lactam spacers can resist infection by the modified 77 phage. This strategy enables the genesis of a population of bacteria that are all re-sensitized and that carry the CRISPR-Cas system. Nevertheless, it may not prove useful in cases where the targets are present in the chromosome or on plasmids carrying toxinantitoxin systems.

One key consideration for engineering moving forward is assessing which class, type and subtype of CRISPR-Cas systems are most lethal and effective, and determining whether it is preferable to co-opt 
endogenous systems and "just" deliver multiplexed self-targeting CRISPR arrays, or co-deliver the CRISPR targeting array and the corresponding effector Cas machinery (Figure 1b). Delivering a CRISPR array alone presents the advantage of requiring a smaller and simpler construct, but the drawback of relying on endogenous Cas proteins which might not be expressed in all conditions in the recipient strains [44]. Once the CRISPR machinery is readily packaged into proper viral vectors, the optimal formulation of bacteriophage preparation will have to be developed and optimized, potentially differentially for each delivery route. Indeed, delivery format is anticipated to vary together with the target organism and site of infection (i.e. intestinal vs. topical vs. lung infections vs. urogenital tract).

\section{Resistance}

An important limitation of all strategies that employ phages as delivery vectors is their host range. Most phages can only infect a limited number of strains within a given species. Host range can be limited by a number of factors [45]. Surface receptors might not be present or can be hidden; entry exclusion systems can block DNA injection in the cell; restriction-modification systems and CRISPRCas systems can degrade the phage DNA; and finally, abortive infection systems can block later stages of the phage cycle by coercing infected cell to commit suicide. With the exception of abortive infection systems which would not be triggered by a synthetic genetic circuit, all these phage defense pathways can also present obstacles to the delivery of CRISPR DNA into bacteria (Figure 1). Phage cocktails have traditionally been used to overcome these hurdles by ensuring that at least one phage is able to infect the target bacteria, but these cocktails can be complex to formulate and produce at industrial scales, and also present additional regulatory challenges [46]. Phages with extended host ranges can also be obtained either through engineering or selection $[47,48]$. In a recent study Qimron and colleagues demonstrate how a T7 phagemid can be used to effectively evolve tails enabling efficient transduction in desired host strains [49].

In addition to resistance mechanisms that block the proper delivery of the CRISPR-Cas system, the CRISPR-Cas system itself can fail in several ways. Mutations in the CRISPR-Cas system or in the target can allow bacteria to survive. In the first case, bacteria would still be sensitive to a functional CRISPRCas system, but in the second case, they would effectively escape. Several studies have shown how large deletions can occur in the target region enabling bacteria to survive $[30,37,50]$. Such outcomes can be seen as positive, as they would result in the loss of antibiotic resistance or virulence traits. Nonetheless, point mutations that preserve the gene function might enable bacteria to escape recognition by the Cas nucleases. This can be solved easily by programming the CRISPR-Cas system to target several positions at the undesired locus simultaneously. Fittingly, CRISPR are by nature arrays that encompass multiple targeting sequences, enabling easy and convenient multiplexing for 
antimicrobials. Finally, anti-CRISPR proteins have recently been characterized in some phages [51]. In future, they might be coopted by bacteria to escape CRISPR antimicrobial therapies.

\section{Perspectives}

CRISPR-Cas systems have been successfully repurposed to target virulence factors and antibiotic resistance genes in bacteria, and constitute an appealing option for programmable and sequencespecific antimicrobials. They can efficiently kill a target population when delivered by phage capsids in vitro and can also reduce the colonization of a target population in vivo. In addition, resistant bacteria can be re-sensitized to an antibiotic by curing plasmids carrying resistance genes. In order to bring these strategies to the clinic, specific indications and therapeutic approaches will have to be established. These therapeutic approaches should also be discussed in comparison to other specific antimicrobials such as phage therapy, antimicrobial peptides, antibodies or vaccines, which was not possible within the framework of this short review. The unique advantage of CRISPR-based antimicrobials over all these other strategies is their ability to kill bacteria based on their sequence. This might prove advantageous in cases where it is desirable to eliminate only a select group of bacteria within a species, something that would be arguably impossible to achieve with incumbent strategies. Of course, CRISPR-based approaches would also address two grand challenges of currently available antibiotics, namely: (1) to prevent the indiscriminate eradication of bacteria that might be beneficial; (2) to lessen the selective pressure for resistance (by allowing the non-target population to thrive and occupy the ecological niche). This opens new avenues for CRISPR-based technologies to control the composition of microbial communities rather than using it as a traditional broadspectrum antibiotic.

\section{Financial and competing interests disclosure}

The authors are inventors on several patents related to various uses of CRISPR-based technologies. $\mathrm{RB}$ is a co-founder and SAB member of Intellia Therapeutics and Locus Biosciences. DB is co-founder and SAB member of Eligo Bioscience.

\section{Acknowledgements}

Funding: This work was supported by the European Research Council (ERC) under the Europe Union's Horizon 2020 research and innovation program (grant agreement No [677823]); the French Government's Investissement d'Avenir program; Laboratoire d'Excellence 'Integrative Biology of Emerging Infectious Diseases' [ANR-10-LABX-62-IBEID]; the Pasteur-Weizmann consortium and funds from NC State University and the North Carolina Ag Foundation. 
A

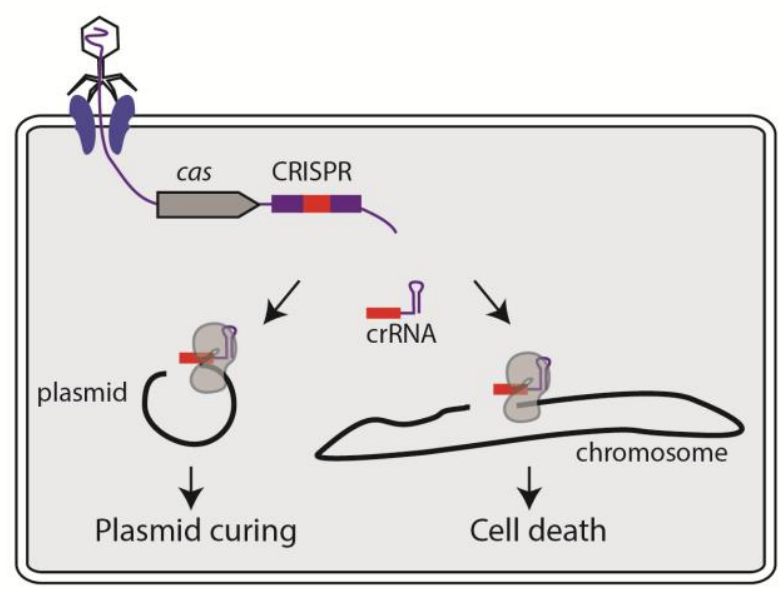

B Endogeneous CRISPR-Cas Exogenous CRISPR-Cas

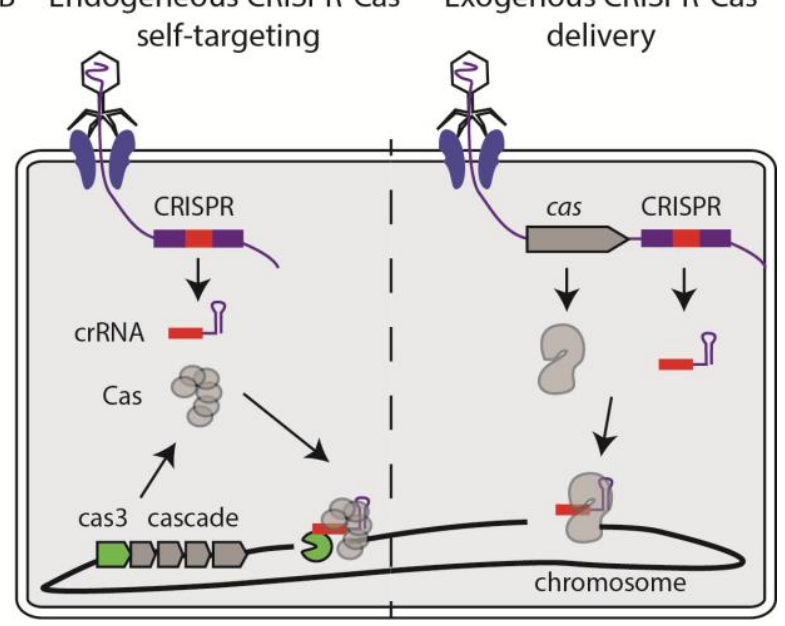

C

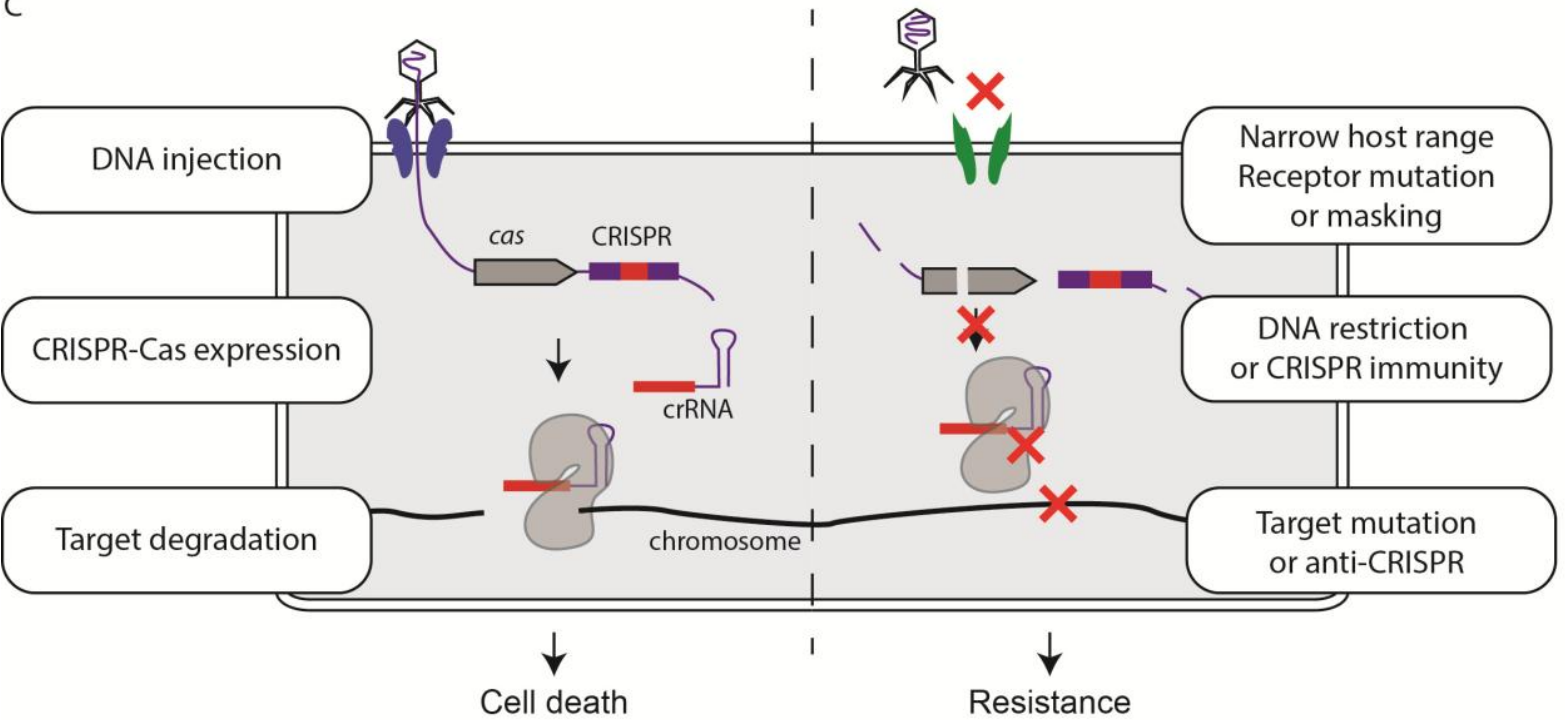

213 Figure 1. CRISPR antimicrobials. A) After injection of a CRISPR system, Cas nuclease cleavage of a

214 target carried by a plasmid leads to plasmid loss while cleavage in the chromosome leads to cell

215 death. B) If the target bacterium carries a endogenous CRISPR-Cas system, one can simply deliver a self-targeting CRISPR array to direct Cas nucleases towards a desired locus. Another strategy is to deliver an exogenous CRISPR-Cas system. C) Summary of CRISPR antimicrobials action and possible resistance mechanisms at every step. The phage vector might not be able to inject its DNA due to narrow host range, receptor mutations or masking. After injection, DNA can be degraded by the action of restriction enzymes or CRISPR-Cas systems. Finally, anti-CRISPR proteins or mutations in the target sequence might block target recognition and cleavage. 


\section{References}

1. Marraffini LA: CRISPR-Cas immunity in prokaryotes. Nature 2015, 526:55-61.

2. Hsu PD, Lander ES, Zhang F: Development and applications of CRISPR-Cas9 for genome engineering. Cell 2014, 157:1262-78.

3. Ledford H: CRISPR, the disruptor. Nat. News 2015, 522:20.

4. Pennisi E: The CRISPR craze. Science 2013, 341:833-6.

5. Makarova KS, Wolf YI, Alkhnbashi OS, Costa F, Shah SA, Saunders SJ, Barrangou R, Brouns SJJ, Charpentier E, Haft DH, et al.: An updated evolutionary classification of CRISPR-Cas systems. Nat. Rev. Microbiol. 2015, 13:722-736.

* This study updated the classification and nomenclature of CRISPR-Cas systems and outlined their occurrence and diversity in bacteria and archaea.

6. Brouns SJ, Jore MM, Lundgren M, Westra ER, Slijkhuis RJ, Snijders AP, Dickman MJ, Makarova $\mathrm{KS}$, Koonin EV, van der Oost J: Small CRISPR RNAs guide antiviral defense in prokaryotes. Science 2008, 321:960-4.

7. Sinkunas T, Gasiunas G, Waghmare SP, Dickman MJ, Barrangou R, Horvath P, Siksnys V: In vitro reconstitution of Cascade-mediated CRISPR immunity in Streptococcus thermophilus. EMBO J 2013, 32:385-94.

8. Garneau JE, Dupuis ME, Villion M, Romero DA, Barrangou R, Boyaval P, Fremaux C, Horvath P, Magadan AH, Moineau S: The CRISPR/Cas bacterial immune system cleaves bacteriophage and plasmid DNA. Nature 2010, 468:67-71.

9. Gasiunas G, Barrangou R, Horvath P, Siksnys V: Cas9-crRNA ribonucleoprotein complex mediates specific DNA cleavage for adaptive immunity in bacteria. Proc. Natl. Acad. Sci. 2012, 109:15539-15540.

10. Jinek M, Chylinski K, Fonfara I, Hauer M, Doudna JA, Charpentier E: A programmable dualRNA-guided DNA endonuclease in adaptive bacterial immunity. Science 2012, 337:816-21.

11. Barrangou R, Doudna JA: Applications of CRISPR technologies in research and beyond. Nat. Biotechnol. 2016, 34:933-941.

12. Citorik RJ, Mimee M, Lu TK: Sequence-specific antimicrobials using efficiently delivered RNA-guided nucleases. Nat Biotechnol 2014, 32:1141-5.

** This report established a proof of concept that CRISPR-Cas9 systems can be delivered by phages to target $\mathrm{E}$. coli in vitro and in vivo.

13. Bikard D, Jiang W, Samai P, Hochschild A, Zhang F, Marraffini LA: Programmable repression and activation of bacterial gene expression using an engineered CRISPR-Cas system. Nucleic Acids Res. 2013, 41:7429-7437.

14. Jiang W, Bikard D, Cox D, Zhang F, Marraffini LA: RNA-guided editing of bacterial genomes using CRISPR-Cas systems. Nat Biotechnol 2013, 31:233-239. 
15. Luo ML, Mullis AS, Leenay RT, Beisel CL: Repurposing endogenous type I CRISPR-Cas systems for programmable gene repression. Nucleic Acids Res. 2015, 43:674-681.

16. Qi LS, Larson MH, Gilbert LA, Doudna JA, Weissman JS, Arkin AP, Lim WA: Repurposing CRISPR as an RNA-guided platform for sequence-specific control of gene expression. Cell 2013, 152:1173-83.

17. Goren M, Yosef I, Qimron U: Sensitizing pathogens to antibiotics using the CRISPR-Cas system. Drug Resist. Updat. Rev. Comment. Antimicrob. Anticancer Chemother. 2017, 30:1-6.

18. Horvath P, Romero DA, Coute-Monvoisin AC, Richards M, Deveau H, Moineau S, Boyaval P, Fremaux C, Barrangou R: Diversity, activity, and evolution of CRISPR loci in Streptococcus thermophilus. J Bacteriol 2008, 190:1401-12.

19. Stern A, Keren L, Wurtzel O, Amitai G, Sorek R: Self-targeting by CRISPR: gene regulation or autoimmunity? Trends Genet 2010, 26:335-40.

20. Semenova E, Jore MM, Datsenko KA, Semenova A, Westra ER, Wanner B, van der Oost J, Brouns SJ, Severinov K: Interference by clustered regularly interspaced short palindromic repeat (CRISPR) RNA is governed by a seed sequence. Proc Natl Acad Sci U A 2011, 108:10098-103.

21. Wiedenheft B, van Duijn E, Bultema JB, Waghmare SP, Zhou K, Barendregt A, Westphal W, Heck AJ, Boekema EJ, Dickman MJ, et al.: RNA-guided complex from a bacterial immune system enhances target recognition through seed sequence interactions. Proc Natl Acad Sci U A 2011, 108:10092-7.

22. Sternberg SH, Redding S, Jinek M, Greene EC, Doudna JA: DNA interrogation by the CRISPR RNA-guided endonuclease Cas9. Nature 2014, 507:62-7.

23. Paez-Espino D, Morovic W, Sun CL, Thomas BC, Ueda K, Stahl B, Barrangou R, Banfield JF: Strong bias in the bacterial CRISPR elements that confer immunity to phage. Nat. Commun. 2013, 4:1430.

24. Datsenko KA, Pougach K, Tikhonov A, Wanner BL, Severinov K, Semenova E: Molecular memory of prior infections activates the CRISPR/Cas adaptive bacterial immunity system. Nat Commun 2012, 3:945.

25. Levy A, Goren MG, Yosef I, Auster O, Manor M, Amitai G, Edgar R, Qimron U, Sorek R: CRISPR adaptation biases explain preference for acquisition of foreign DNA. Nature 2015, 520:505-510.

26. Modell JW, Jiang W, Marraffini LA: CRISPR-Cas systems exploit viral DNA injection to establish and maintain adaptive immunity. Nature 2017, 544:101-104.

27. Staals RHJ, Jackson SA, Biswas A, Brouns SJJ, Brown CM, Fineran PC: Interference-driven spacer acquisition is dominant over naive and primed adaptation in a native CRISPR-Cas system. Nat. Commun. 2016, 7:12853.

28. Edgar R, Qimron U: The Escherichia coli CRISPR system protects from lambda lysogenization, lysogens, and prophage induction. J Bacteriol 2010, 192:6291-4.

* This is the first report of artificial self targeting by a CRISPR system 
29. Gomaa AA, Klumpe HE, Luo ML, Selle K, Barrangou R, Beisel CL: Programmable removal of bacterial strains by use of genome-targeting CRISPR-Cas systems. MBio 2013, 5:e00928-13.

** This study showed that endogenous CRISPR-Cas systems can be repurposed to generate programmable cell death in bacteria and selectively target different genotypes in a mixed population.

30. Vercoe RB, Chang JT, Dy RL, Taylor C, Gristwood T, Clulow JS, Richter C, Przybilski R, Pitman $A R$, Fineran PC: Cytotoxic chromosomal targeting by CRISPR/Cas systems can reshape bacterial genomes and expel or remodel pathogenicity islands. PLoS Genet 2013, 9:e1003454.

* This early report revealed the lethality and some genomic outcomes of self-targeting by CRISPR-Cas systems.

31. Westra ER, van Erp PB, Kunne T, Wong SP, Staals RH, Seegers CL, Bollen S, Jore MM, Semenova $\mathrm{E}$, Severinov $\mathrm{K}$, et al.: CRISPR immunity relies on the consecutive binding and degradation of negatively supercoiled invader DNA by Cascade and Cas3. Mol Cell 2012, 46:595605.

32. Caliando BJ, Voigt CA: Targeted DNA degradation using a CRISPR device stably carried in the host genome. Nat. Commun. 2015, 6:6989.

33. Bikard D, Hatoum-Aslan A, Mucida D, Marraffini LA: CRISPR interference can prevent natural transformation and virulence acquisition during in vivo bacterial infection. Cell Host Microbe 2012, 12:177-186.

34. Beloglazova N, Petit P, Flick R, Brown G, Savchenko A, Yakunin AF: Structure and activity of the Cas3 HD nuclease MJ0384, an effector enzyme of the CRISPR interference. EMBO J. 2011, 30:4616-4627.

35. Sinkunas T, Gasiunas G, Fremaux C, Barrangou R, Horvath P, Siksnys V: Cas3 is a singlestranded DNA nuclease and ATP-dependent helicase in the CRISPR/Cas immune system. EMBO J 2011, 30:1335-42.

36. Wigley DB: Bacterial DNA repair: recent insights into the mechanism of $\operatorname{RecBCD}, A d d A B$ and AdnAB. Nat. Rev. Microbiol. 2013, 11:9-13.

37. Cui L, Bikard D: Consequences of Cas9 cleavage in the chromosome of Escherichia coli. Nucleic Acids Res. 2016, 44:4243-4251.

* This study investigated the outcomes of Cas9-based self-targeting in bacteria and established the interplay between DNA damage and DNA repair.

38. Hagens S, Bläsi U: Genetically modified filamentous phage as bactericidal agents: a pilot study. Lett. Appl. Microbiol. 2003, 37:318-323.

39. Moradpour Z, Sepehrizadeh Z, Rahbarizadeh F, Ghasemian A, Yazdi MT, Shahverdi AR: Genetically engineered phage harbouring the lethal catabolite gene activator protein gene with an inducer-independent promoter for biocontrol of Escherichia coli. FEMS Microbiol. Lett. 2009, 296:67-71.

40. Westwater C, Kasman LM, Schofield DA, Werner PA, Dolan JW, Schmidt MG, Norris JS: Use of Genetically Engineered Phage To Deliver Antimicrobial Agents to Bacteria: an Alternative Therapy for Treatment of Bacterial Infections. Antimicrob. Agents Chemother. 2003, 47:1301-1307. 
41. Hagens S, Habel A, Ahsen U von, Gabain A von, Bläsi U: Therapy of Experimental Pseudomonas Infections with a Nonreplicating Genetically Modified Phage. Antimicrob. Agents Chemother. 2004, 48:3817-3822.

42. Bikard D, Euler CW, Jiang W, Nussenzweig PM, Goldberg GW, Duportet X, Fischetti VA, Marraffini LA: Exploiting CRISPR-Cas nucleases to produce sequence-specific antimicrobials. Nat Biotechnol 2014, 32:1146-50.

** This report established a proof of concept that CRISPR-Cas9 systems can be delivered by phages to target $\mathrm{S}$. aureus in vitro and in vivo.

43. Yosef I, Manor M, Kiro R, Qimron U: Temperate and lytic bacteriophages programmed to sensitize and kill antibiotic-resistant bacteria. Proc. Natl. Acad. Sci. 2015, 112:7267-7272.

** This study provides novel strategies to re-sensitize bacteria to antibiotics using plasmid targeting CRISPR-Cas systems.

44. Westra ER, Pul Ü, Heidrich N, Jore MM, Lundgren M, Stratmann T, Wurm R, Raine A, Mescher $\mathrm{M}$, Van Heereveld $\mathrm{L}$, et al.: H-NS-mediated repression of CRISPR-based immunity in Escherichia coli K12 can be relieved by the transcription activator LeuO. Mol. Microbiol. 2010, 77:1380-1393.

45. Labrie SJ, Samson JE, Moineau S: Bacteriophage resistance mechanisms. Nat Rev Microbiol 2010, 8:317-27.

46. Sulakvelidze A, Alavidze Z, Morris JG: Bacteriophage Therapy. Antimicrob. Agents Chemother. 2001, 45:649-659.

47. Ando H, Lemire S, Pires DP, Lu TK: Engineering Modular Viral Scaffolds for Targeted Bacterial Population Editing. Cell Syst. 2015, 1:187-196.

48. Mapes AC, Trautner BW, Liao KS, Ramig RF: Development of expanded host range phage active on biofilms of multi-drug resistant Pseudomonas aeruginosa. Bacteriophage 2016, 6:e1096995.

49. Yosef I, Goren MG, Globus R, Molshanski-Mor S, Qimron U: Extending the Host Range of Bacteriophage Particles for DNA Transduction. Mol. Cell 2017, 66:721-728.e3.

50. Selle K, Klaenhammer TR, Barrangou R: CRISPR-based screening of genomic island excision events in bacteria. Proc. Natl. Acad. Sci. 2015, 112:8076-8081.

* This report established that native CRISPR-Cas systems can be co-opted to select for rare deletion events of expendable genetic islands.

51. Pawluk A, Staals RHJ, Taylor C, Watson BNJ, Saha S, Fineran PC, Maxwell KL, Davidson AR: Inactivation of CRISPR-Cas systems by anti-CRISPR proteins in diverse bacterial species. Nat. Microbiol. 2016, 1:16085. 